\title{
Nicht jeder Husten braucht ein Antibiotikum
}

— Obwohl Atemwegsinfekte meist viral bedingt sind, werden dafür nach wie vor sehr häufig Antibiotika verschrieben. Vielfach setzen Ärzte voraus, dass ihre Patienten eine Antibiotikaverordnung erwarten. Eine aktuelle Befragung von Patienten zeigt jedoch eindrucksvoll, dass dies bei weitem nicht so oft der Fall ist, wie die Ärzte das vermuten [Faber M S et al. Eurosurveill 2010 (35); 15: 1-7]. In der Studie des Robert-Koch-Instituts und der Charité Berlin wurden 1.076 Patienten zu ihrer Einstellung zu Antibiotika befragt: Über 90\% der Befragten gaben an, Antibiotika nur zu nehmen, wenn es ihnen absolut notwendig erscheint, und nur 10\% erwarten bei einer Erkältung die Verschreibung eines Antibiotikums. Den Arzt wechseln, wenn sie kein Antibiotikum bekämen, würden nur 3\% der Befragten.

Entscheidend für die Zufriedenheit der Mehrzahl der Erkältungspatienten ist eine wirksame nicht antibiotische Therapie, wie sie das Phytotherapeutikum Bronchipret ${ }^{\oplus}$ bietet. Aufgrund der überzeugenden Ergebnisse zweier plazebokontrollierter, randomisierter, doppelblinder klinischer Studien empfiehlt die Deutsche Gesellschaft für Pneumologie und Beatmungsmedizin das Kombinationspräparat in beiden Darreichungsformen (Saft und Tabletten) als einziges Therapeutikum gegen akuten Husten in den Leitlinien zur „Diagnostik und Therapie von erwachsenen Patienten mit akutem und chronischem Husten“. Das Präparat reduziert die Hustenfrequenz im Vergleich zu Plazebo signifikant, halbiert die Zahl der Hustenanfälle um zwei Tage schneller als Plazebo und ist auch in weiteren Parametern, wie z.B. hustenbedingten Schlafstörungen, Plazebo überlegen.

Eine Übertragbarkeit der Studienergebnisse auf andere Phytotherapeutika ist laut HustenLeitlinie nicht möglich, da die Wirksamkeit pflanzlicher Medikamente wesentlich von der Qualität der Pflanzen, der Methodik und
Standardisierung der Extraktion und dem Herstellungsverfahren abhängt.

Nach Informationen von Bionorica SE

Ärzte vermuten oft, ihre Patienten wollen auch bei banalen Erkältungen partout ein Antibiotikum - und irren sich damit häufig.

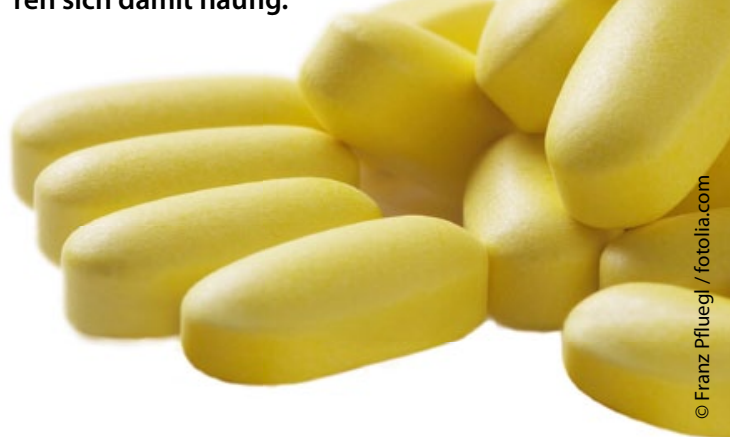

sich ein Großteil der befragten Patienten bei folgenden Tätigkeiten beeinträchtigt: 93\% bei täglichen Aktivitäten, Sport und Freizeit, $86 \%$ im Beruf und in der Schule, $80 \%$ der Patienten beklagen Schlafprobleme bzw. fühlen sich durch körperliche Symptome beeinträchtigt ( $83 \%)$. Besonders stark litten die Patienten an einer laufenden bzw. verstopften Nase. Bei den Augen wurde vor allem das Jucken als belastend empfunden.

Auf eine spezifische Immuntherapie angesprochen, die aus vier subkutanen Injektionen besteht und die Allergiebeschwerden um 35\% vermindert, hielt eine überwiegende Mehrheit der 412 diesbezüglich befragten Patienten diese mögliche Verbesserung für wichtig: $96 \%$ dieser Patienten bewerteten eine Verbesserung ihrer Beschwerden um 35\% als bedeutsam, $60 \%$ sogar als sehr wichtig.

Die Umfrage zeigt: Allergiepatienten fühlen sich trotz symptomatischer Medikation insbesondere im beruflichen und sozialen Leben so sehr eingeschränkt, dass jede positive Veränderung als spürbare Verbesserung der Lebensqualität empfunden wird.

$g z$

Nach Informationen von Bencard Allergie 